\title{
Robust Segmentation and Anatomical Labeling of the Airway Tree from Thoracic CT Scans
}

\author{
Bram van Ginneken, Wouter Baggerman, and Eva M. van Rikxoort \\ Image Sciences Institute, University Medical Center Utrecht, The Netherlands
}

\begin{abstract}
A method for automatic extraction and labeling of the airway tree from thoracic CT scans is presented and extensively evaluated on 150 scans of clinical dose, low dose and ultra-low dose data, in inspiration and expiration from both relatively healthy and severely ill patients. The method uses adaptive thresholds while growing the airways and it is shown that this strategy leads to a substantial increase in the number, total length and number of correctly labeled airways extracted. From inspiration scans on average 170 branches are found, from expiration scans 59 .
\end{abstract}

\section{Introduction}

Multi-slice CT scanning technology has revolutionized the in vivo study of the lungs and motivates the need for pulmonary image analysis [1. Automated extraction and labeling of the bronchial tree from thoracic CT scans is vital to accurately quantify airway morphology which is increasingly used to measure progression and response to treatment for a variety diseases. Another important application is computer-assisted bronchoscopy.

A wide variety of methods have been developed to segment the airways 2345678910 11. Some of these include or focus specifically on anatomical labeling of airway segments 21213. Most methods have been evaluated on a small number of scans. Evaluation on low-dose scans is rare (14] is an exception), as are application to expiration scans and scans with substantial pathology.

In this work, a method is proposed to segment the complete airway tree. Our approach is based on the generic tree extraction framework outlined in 46 and introduces several modifications and new rules for accepting segments. A key contribution is the introduction of a multi-threshold approach to increase robustness. Furthermore, a novel algorithm for anatomical labeling is presented.

Extensive results are presented from 150 scans that include clinical dose, low dose and ultra low-dose, inspiration and expiration scans and data from asymptomatic subjects as well as interstitial lung disease patients with massive pathology.

\section{Method}

The backbone of our algorithm is an implementation of the framework given in 46]. In this section we discuss the initialization, briefly review the framework, describe

D. Metaxas et al. (Eds.): MICCAI 2008, Part I, LNCS 5241, pp. 219-226, 2008.

(C) Springer-Verlag Berlin Heidelberg 2008 
our rules for accepting voxels, fronts and segments, introduce the multi-threshold extension of the method and finally describe the anatomical labeling algorithm.

Initialization. The trachea and the lungs are automatically segmented with a method based on [1516]. Central dark circular regions are searched to find a start point in the trachea, followed by region growing with multiple optimal thresholds to extract the trachea and the lungs. The lung segmentation is used to infer the scan orientation. From the trachea segmentation a seed point is determined in the axial slice that contains the center of gravity of the structure. Only growing in the basal direction is allowed.

Tree segmentation framework. The segments of the bronchial tree are obtained by wavefront propagation. The initial seed point provides the first front. At every iteration, all unprocessed voxels connected to the front that satisfy the voxel criteria form the new wavefront. The segment is allowed to keep growing when the front meets the wavefront criteria. If the new front consists of multiple parts, a segment is complete and accepted if it complies with the segment criteria. To avoid spurious front splittings due to noise, a large 80-connectivity value is used to detect them. New fronts are pushed on a stack and the next front from the stack is propagated. The algorithm terminates when the stack is empty. While the fronts propagate, the centerline or skeleton of the tree and the local segment diameter are computed and this information is used in several of the acceptance criteria. An important difference with 46] is that we use region growing to obtain the new front. To avoid diamond- or cuboid-shaped fronts, growing is restricted to within a sphere from the last calculated center point with a diameter slightly larger than the last calculated segment diameter.

Rules for accepting voxels, wavefronts and segments. Voxels are accepted when their density (in Hounsfield Units or HU) is below a threshold $t$, or (to be less sensitive to noise) the HU value in a $3 \times 3 \times 3$ neighborhood around the voxel $i s<t$. For every new front, three checks are applied to the segment grown so far, and if they are violated the entire segment is removed. First, the segment's current radius must be smaller than 1.5 times the minimum radius found in any parent segment. This ensures that diameters of bronchi diminish. When leaking occurs, this rule is typically violated. Second, a front is not allowed to touch any other segment (segments are grown in a breadth first fashion). Third, the length of the segment should not be more than 5 times its radius. This ensures that partly grown segments are accepted before a leakage occurs that could discard a large part of an airway. A completed segment is only accepted if it meets two more requirements: The angle it makes with its parent should be $<100^{\circ}$ and the average ratio of radii of two consecutive fronts should not exceed 1.1. The latter check ensures that the segment is not widening, which typically indicates leakage.

Postprocessing. After the bronchial tree has been extracted, several postprocessing steps are performed. First all minor trailing segments (i.e. segments without descendants) are removed. Segments are considered minor if their length is smaller than $3 \mathrm{~mm}$ and their volume is below $25 \mathrm{~mm}^{3}$. Next, the tree structure 


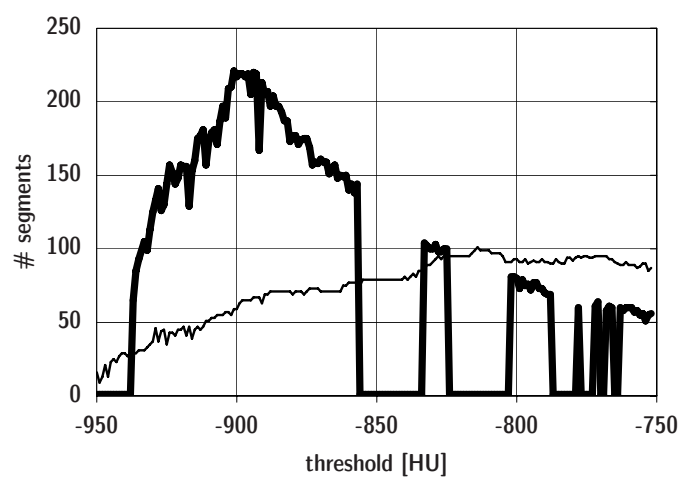

Fig. 1. The total number of segments found in the airway tree as a function of the threshold $t$ for voxel acceptance for two scans. The thick line demonstrates that the results can be sensitive to small changes in $t$. For both cases, the multi-threshold method found many more segments: 289 and 181 for the thick and thin line, respectively.

is scanned for segments that have exactly one descendant, and these segments are merged. Finally, holes in the segments, primarily caused by noise, are filled. Adaptive acceptance rules. We have observed that with these rules for accepting wavefronts and segments, leakage into the parenchyma is virtually impossible to occur, and thus all segments found are true airways. Not all airways are found, however, and in fact it is possible that large parts of the airway tree are missed. Quite often, small changes in the value for the voxel acceptance threshold $t$, have a profound effect on the number and total length of detected airways. Note that it is not the case that higher values for $t$ automatically lead to more voxels considered airways. Surely, more voxels are accepted when computing a new front when $t$ is increased, but these fronts or these segments may subsequently be rejected by the front and segment acceptance rules. The algorithm thus manifests a complex interplay between the rules at various levels. The effect is illustrated in Fig. 1, This figure also shows that the optimal value for $t$ varies from scan to scan. For most scans, $t=-900$ gives the best results, so this value was used in what we refer to as the single threshold method.

To overcome these limitations and obtain a more robust segmentation that includes as many peripheral airways as possible, we propose to make the process adaptive. Every segment is first grown with a high threshold $t=-750$. If rejected, the segment is regrown with a lower threshold $t+k \Delta t$ with $k=1, \ldots, 18$ and $\Delta t=-10$. This is referred to as the multi-threshold method. It renders the extraction process adaptive: at every position in the scan the maximum number of airway voxels are selected while the front and segment rules still ensure that no leaking can occur.

Anatomical labeling. After the tree has been segmented, anatomical labels for branches up to the segmental level are automatically assigned. We use 32 distinct labels following the nomenclature from [12]. The fact that trifurcations are usually two consecutive bifurcations lead us to define five small intermediate segments that may or may not be present. 
Table 1. Average number and total length (in $\mathrm{mm}$ ) of all airways per generation, per scan, split over the three databases

\begin{tabular}{|c|c|c|c|c|c|c|c|c|c|c|c|c|}
\hline & \multicolumn{4}{|c|}{ Database 1} & \multicolumn{4}{|c|}{ Database 2} & \multicolumn{4}{|c|}{ Database 3} \\
\hline & & gle & & ulti & & agle & & ulti & & ngle & & ulti \\
\hline en. & \# & gin & $\#$ & gth & \# & gth & $\# 1$ & gth & $\#$ & gth & $\# 1$ & igth \\
\hline 1 & 2 & 97 & 2 & 99 & 2 & 92 & 2 & 96 & 2 & 88 & 2 & 88 \\
\hline 2 & 4 & s & 4 & 68 & & 64 & 4 & 70 & & 58 & 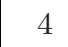 & $5 s$ \\
\hline 3 & 8 & 102 & 8 & 100 & 7 & 85 & 8 & 103 & & 88 & 8 & 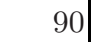 \\
\hline 4 & 14 & 188 & 16 & 214 & 7 & 87 & 13 & 162 & 11 & 141 & 14 & 163 \\
\hline 5 & 17 & 251 & 27 & 378 & & 50 & 14 & 179 & & 206 & 22 & 29 \\
\hline 6 & 14 & 193 & 35 & 430 & 2 & 28 & 8 & 85 & 1 & 178 & 32 & 366 \\
\hline 7 & 10 & 140 & 27 & 330 & 1 & 17 & 5 & 54 & 10 & 130 & 29 & 29 \\
\hline 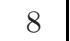 & - & 8 & 18 & 218 & 1 & 9 & 2 & 29 & & 83 & 21 & 20 \\
\hline 9 & 4 & 62 & 11 & 143 & 0 & إ & 1 & 10 & & 57 & 15 & 15 \\
\hline 10 & 2 & 30 & 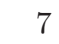 & 92 & 0 & 1 & 0 & 2 & 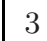 & 35 & 10 & 100 \\
\hline 1 & 1 & 13 & 4 & 55 & 0 & 0 & 0 & 0 & 2 & 16 & 10 & 61 \\
\hline$>11$ & 1 & 11 & 5 & 56 & 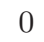 & 0 & 0 & 0 & 2 & 17 & 10 & \\
\hline all & 81 & 1238 & 166 & 2183 & 30 & 434 & 59 & 789 & 82 & 1096 & 174 & 194 \\
\hline
\end{tabular}

To assign the labels, the segment labels of 17 trees were manually assigned. The distribution of several segment characteristics were studied, and orientation, average radius, and angle with the parent segment were found most discriminative. Assuming independent normal distributions for these characteristics over the segments, we can measure a probability that a particular label should be assigned to a test segment. To assign the actual label, we look at the probability of the segment conditioned on those of its children and grand children. Labels are assigned in a recursive process, starting at the trachea, which is known from the initial seed point. At every step all unlabeled segments with a labeled parent are considered and the most probable labels are assigned.

\section{$3 \quad$ Experimental Results}

Experiments have been performed on three databases, each holding 50 scans from 16-slice scanners or higher, with sub-millimeter near isotropic resolution. Database 1 contains low-dose $\left(\mathrm{CTDI}_{\mathrm{vol}} \approx 3 \mathrm{mGy}\right)$ scans from a lung cancer screening program from asymptomatic subjects, scanned at full inspiration. Database 2 contains scans from the same subjects, now scanned at ultra-low-dose $\left(\mathrm{CTDI}_{\mathrm{vol}} \approx 1\right.$ mGy) at end-expiration. Database 3 consists of scans from patients with interstitial lung disease with severe abnormalities present in the scans, all acquired at full inspiration and with a clinical dose $\left(\mathrm{CTDI}_{\mathrm{vol}} \approx 10 \mathrm{mGy}\right)$.

We applied both the single and the multi-threshold method to all 150 scans. Table 1 reports the total number of segments found and their length, per generation. Table 2 list for each lobar and segmental branch how often is was detected. Fig. 2 compares the results of the single and multi-threshold method in a 
Table 2. For each of the 32 labeled segments in the bronchial tree, the percentages of extraction are listed for each database and for the single threshold method $(\mathrm{S})$ and the multi-threshold method (M)

Database 1 Database 2 Database 3

\begin{tabular}{|c|c|c|c|c|c|c|}
\hline & $\mathrm{S}$ & M & $\mathrm{S}$ & M & $\mathrm{S}$ & M \\
\hline Trachea & 100 & $\overline{100}$ & 100 & $\overline{100}$ & 100 & 100 \\
\hline RMB & 98 & 100 & 100 & 100 & 92 & 96 \\
\hline RUL & 96 & 98 & 98 & 100 & 92 & 94 \\
\hline RB1 & 96 & 98 & 68 & 94 & 82 & 88 \\
\hline RB2 & 92 & 96 & 72 & 94 & 84 & 86 \\
\hline RB3 & 94 & 98 & 80 & 96 & 86 & 88 \\
\hline BronInt & 96 & 98 & 98 & 100 & 92 & 94 \\
\hline $\mathrm{RB} 4+5$ & 96 & 98 & 86 & 98 & 92 & 94 \\
\hline RB4 & 74 & 96 & 58 & 88 & 74 & 90 \\
\hline RB5 & 74 & 96 & 58 & 88 & 74 & 90 \\
\hline RB6 & 96 & 98 & 86 & 98 & 92 & 94 \\
\hline RLL7 & 96 & 98 & 72 & 98 & 82 & 92 \\
\hline RB7 & 94 & 98 & 62 & 94 & 80 & 88 \\
\hline RLL & 94 & 98 & 62 & 94 & 80 & 88 \\
\hline RB8 & 84 & 98 & 34 & 80 & 62 & 78 \\
\hline RB9 & 84 & 96 & 40 & 80 & 58 & 80 \\
\hline RB10 & 84 & 98 & 36 & 80 & 60 & 78 \\
\hline LMB & 98 & 100 & 100 & 100 & 92 & 96 \\
\hline LUL & 96 & 100 & 94 & 98 & 92 & 92 \\
\hline $\mathrm{LB} 1+2$ & 92 & 98 & 76 & 96 & 82 & 92 \\
\hline LB1 & 82 & 96 & 54 & 88 & 68 & 88 \\
\hline LB2 & 82 & 96 & 54 & 88 & 68 & 88 \\
\hline LB3 & 94 & 98 & 40 & 86 & 86 & 88 \\
\hline $\mathrm{LB} 4+5$ & 94 & 98 & 90 & 98 & 84 & 90 \\
\hline LB4 & 80 & 96 & 42 & 74 & 74 & 88 \\
\hline LB5 & 80 & 96 & 42 & 74 & 74 & 88 \\
\hline LLB6 & 96 & 100 & 94 & 98 & 92 & 92 \\
\hline LB6 & 96 & 98 & 74 & 96 & 84 & 90 \\
\hline LLB & 96 & 98 & 74 & 96 & 84 & 90 \\
\hline LB8 & 88 & 98 & 40 & 82 & 76 & 88 \\
\hline LB9 & 92 & 98 & 44 & 88 & 76 & 88 \\
\hline LB10 & 88 & 98 & 38 & 84 & 70 & 86 \\
\hline Overall average & $\overline{91}$ & $\overline{98}$ & 68 & $\overline{92}$ & 81 & 90 \\
\hline
\end{tabular}

scatterplot. Fig. 3 shows results for Database 1 (low-dose inspiration) versus the most challenging data used in this study, Database 2 (ultra-low dose expiration).

We have evaluated the accuracy of the labeling by asking two trained human observers to click points in each of the 32 segments. In a preliminary study on 36 scans we found that for the branches detected by the multi-threshold algorithm (98, 92 and $90 \%$, for the three databases, respectively) $90 \%$ were correctly labeled. Most errors were caused by anatomical variations in the airway trees. 

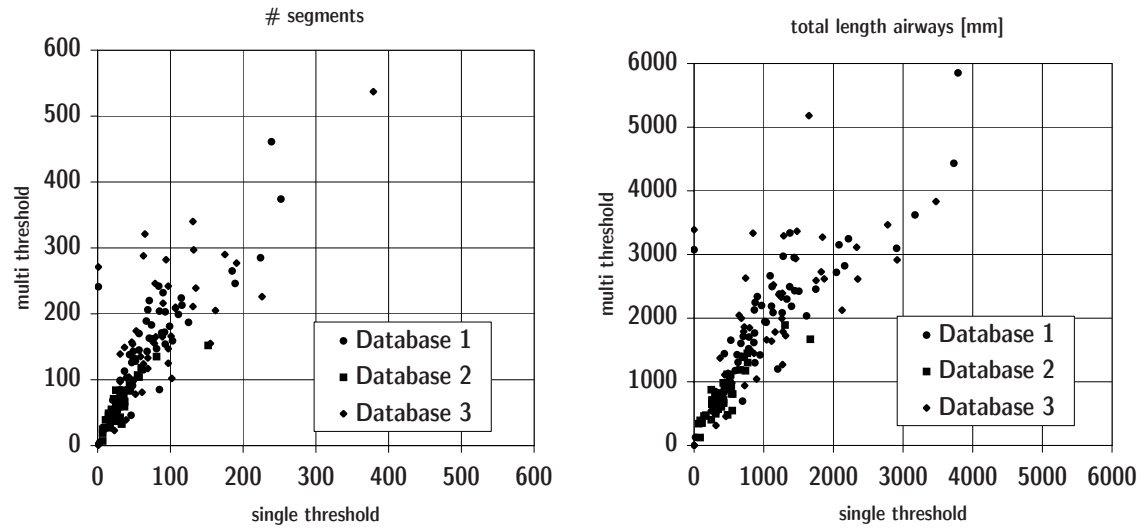

Fig. 2. Scatterplots showing number of segments (left) and total length of all airways in $\mathrm{mm}$ (right) found with the single and multi-threshold method. Each point represents one scan.
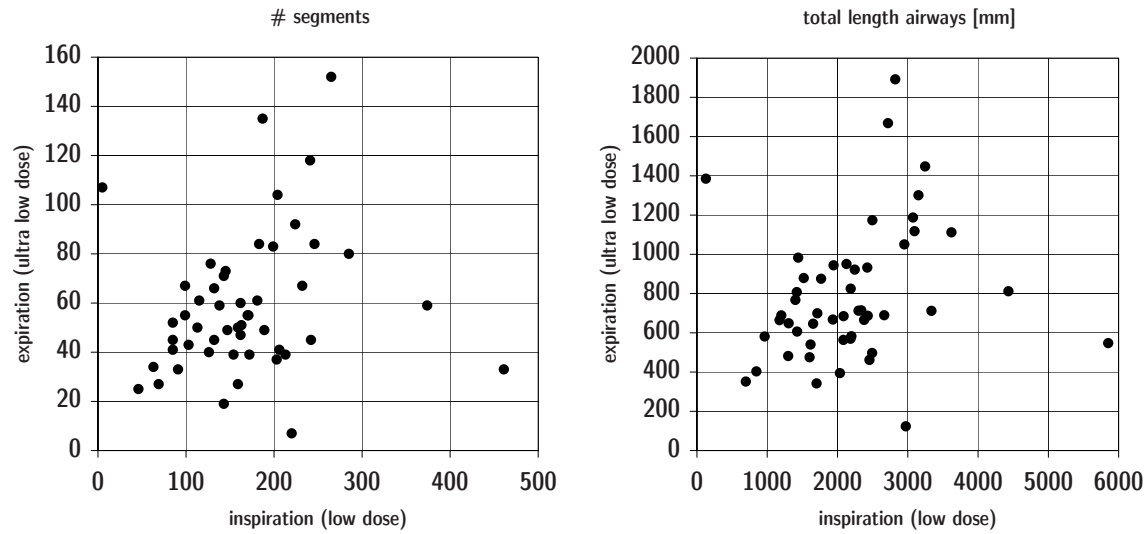

Fig. 3. Scatterplots showing number of segments (left) and total length of all airways in $\mathrm{mm}$ (right) found for the 50 scans in Database 1 (low dose, inspiration scans of asymptomatic subjects) versus the 50 scans in Database 2 (same subjects, scanned with ultra low dose in expiration). Each point represents one scan. Note the different scales on horizontal and vertical axis.

\section{Discussion and Conclusion}

The multi-threshold method effectively uses different HU thresholds for different parts of the bronchial tree. This appears very effective to avoid leakage, and thus early termination of the growth process, which we believe to be the main limitation of the single threshold strategy and previous methods that follow this approach 4614. Those methods already substantially outperform methods that do not employ the higher level rules at segmental level, like explosion control 
region growing. An average number of 170 airways detected in inspiration scans is impressive compared to results reported in other studies. Table 2 shows that the multi-threshold method is capable of extracting the bronchial tree up to segmental level quite robustly, and that its results are substantially better than those of the single threshold method. Both methods produce, by design, almost no false positive findings (incorrect branches). They are also fast, even the multithreshold growing process requires only around 10 seconds on a single-core PC. The labeling takes less than two seconds.

A limitation of using variable thresholds is that the airway diameter estimation may not be consistent throughout the tree. We believe the current system should be followed by an additional step in which the airway lumen and wall are precisely determined.

In all scans we have inspected, there were very small peripheral bronchi present that were not extracted. A specific search for more distal airways and a mechanism to connect these to the tree [7]0 might improve performance even more. Note that such schemes require vastly more computation time. Another useful extension would be to use more elaborate rules for accepting voxels, fronts and segments, based on more complex image information and statistical analysis, as was recently proposed in 11 .

The evaluation of the anatomical labeling is not based on the entire test set and thus preliminary. The accuracy of the procedure could be improved if the possibility of identifying the main anatomical variations in the airway tree up to segmental level were included in the labeling algorithm. Nevertheless it is clear that the majority of labels assigned by our method is correct.

Comparison of the results from Database 1 and 2 shows that the extraction of airways from expiration scans is substantially more difficult, but still generally feasible for the first few generations of the airway tree. The minor differences between Database 1 and 3 show that the proposed method is largely robust to pathology. It is also noteworthy that the total amount and length of airways extracted varies strongly from scan to scan. This makes it clear that comparing results from different methods obtained on different scans is tricky. It would be very useful to compare the different approaches on a single common database.

In conclusion, we have presented a fast, fully automatic method to extract the bronchial tree far into the periphery of the lungs and in addition label the most important branches. By locally adapting the threshold for accepting voxels as being airway, the algorithm is able to grow into airways with thin walls or absent walls at bifurcations, without leaking into the lung parenchyma. Extensive experiments have demonstrated that the method is robust and effective in finding a large number of airways up to ten generations or more.

\section{References}

1. Sluimer, I.C., Schilham, A., Prokop, M., van Ginneken, B.: Computer analysis of computed tomography scans of the lung: a survey. IEEE Trans. Med. Im. 25(4), 385-405 (2006) 
2. Mori, K., Hasegawa, J., Suenaga, Y., Toriwaki, J.: Automated anatomical labeling of the bronchial branch and its application to the virtual bronchoscopy system. IEEE Trans. Med. Im. 19(2), 103-114 (2000)

3. Swift, R.D., Kiraly, A.P., Sherbondy, A.J., Austin, A.L., Hoffman, E.A., McLennan, G., Higgins, W.E.: Automatic axis generation for virtual bronchoscopic assessment of major airway obstructions. Comp. Med. Imag. \& Graph 26, 103-118 (2002)

4. Schlathölter, T., Lorenz, C., Carlsen, I.C., Renisch, S., Deschamps, T.: Simultaneous segmentation and tree reconstruction of the airways for virtual bronchoscopy. In: Proc. SPIE, vol. 4684, pp. 103-113 (2002)

5. Kiraly, A.P., Helferty, J.P., Hoffman, E.A., McLennan, G., Higgins, W.E.: Threedimensional path planning for virtual bronchoscopy. IEEE Trans Med. Im. 23(11), 1365-1379 (2004)

6. Bülow, T., Lorenz, C., Renisch, S.: A general framework for tree segmentation and reconstruction from medical volume data. In: Barillot, C., Haynor, D.R., Hellier, P. (eds.) MICCAI 2004. LNCS, vol. 3216, pp. 533-540. Springer, Heidelberg (2004)

7. Fetita, C.I., Prêteux, F., Beigelman-Aubry, C., Grenier, P.: Pulmonary airways: 3$\mathrm{D}$ reconstruction from multislice CT and clinical investigation. IEEE Trans. Med. Im. 23(11), 1353-1364 (2004)

8. Tschirren, J., Hoffman, E.A., McLennan, G., Sonka, M.: Intrathoracic airway trees: segmentation and airway morphology analysis from low-dose CT scans. IEEE Trans. Med. Im. 24(12), 1529-1539 (2005)

9. Palágyi, K., Tschirren, J., Hoffman, E.A., Sonka, M.: Quantitative analysis of pulmonary airway tree structures. Comput. Biol. Med. 36(9), 974-996 (2006)

10. Higgins, W.E., Graham, M.W., Gibbs, J.D.: Robust system for human airway tree segmentation. In: Proc. SPIE, vol. 6914 (2008)

11. Lo, P., de Bruijne, M.: Voxel classification-based airway tree segmentation. In: Proc. SPIE, vol. 6914 (2008)

12. Tschirren, J., McLennan, G., Palágyi, K., Hoffman, E.A., Sonka, M.: Matching and anatomical labeling of human airway tree. IEEE Trans. Med. Im. 24, 1540-1547 (2005)

13. Bülow, T., Lorenz, C., Wiemker, R., Honko, J.: Point based methods for automatic bronchial tree matching and labeling. In: Proc. SPIE, vol. 6143 (2006)

14. Wiemker, R., Ekin, A., Opfer, R., Bülow, T., Rogalla, P.: Unsupervised extraction and quantification of the bronchial tree on ultra-low-dose vs. standard-dose CT. In: Proc. SPIE, vol. 6143 (2006)

15. Hu, S., Hoffman, E.A., Reinhardt, J.M.: Automatic lung segmentation for accurate quantitation of volumetric X-ray CT images. IEEE Trans. Med. Im. 20(6), 490-498 (2001)

16. Sluimer, I.C., Prokop, M., van Ginneken, B.: Towards automated segmentation of the pathological lung in CT. IEEE Trans. Med. Im. 24(8), 1025-1038 (2005) 